Journal

Natural Product Research >

Formerly Natural Product Letters

Volume 33, 2019 - Issue 1

1442

0

Views CrossRef citations to date Altmetric

Research Articles

\title{
A new sulphated flavone and other phytoconstituents from the leaves of Tetracera indica Merr. and their alpha- glucosidase inhibitory activity
}

Alhassan Muhammad Alhassan, Qamar Uddin Ahmed $\boldsymbol{\nabla}$, Jalifah Latip \& Syed Adnan Ali Shah

Pages 1-8 | Received 24 Oct 2017, Accepted 02 Feb 2018, Published online: 08 Feb 2018

SG Download citation

凤 https://doi-org.ezproxy.um.edu.my/10.1080/14786419.2018.1437427

A) Check for updates

Select Language $\mid \nabla$

Translator disclaimer

\section{Abstract}

The bioactivity guided fractionation of Tetracera indica leaves crude ethanolic extract has afforded the isolation and characterization of six compounds including a new natural product viz., 5,7-dihydroxyflavone-O-8-sulphate (1) and five known flavonoids (2-6). The structures of the compounds were elucidated using 1D and 2D NMR and

HRESIMS spectroscopic analyses. All the isolated compounds were evaluated for their in vitro inhibitory activity against alpha-glucosidase. Compound 1, 5 and $\mathbf{6}$ showed 
strong alpha-glucosidase inhibitory activity, $\mathbf{3}$ and $\mathbf{4}$ displayed weak activity while compound $\mathbf{2}$ was inactive. The interactions of the active compounds with alphaglucosidase were further investigated using molecular docking to confirm their antidiabetic potential.
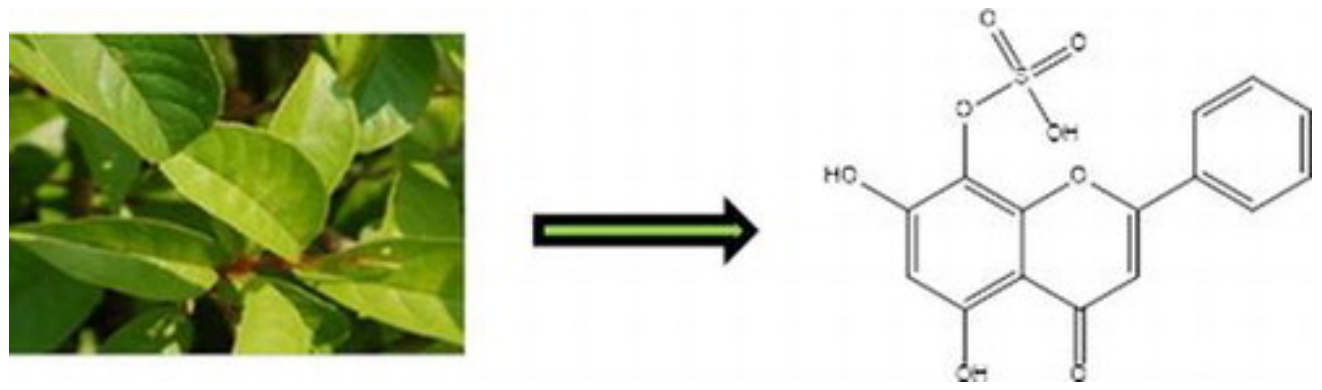

Keywords: Tetracera indica, dilleniaceae, sulphated flavonoid, alpha-glucosidase inhibitory activity, docking 
$>\log$ in

$>$ Shibboleth

$>$ OpenAthens

\section{Restore content access}

$>$ Restore content access for purchases made as guest

\section{Purchase *}

\section{Online}

Article Purchase

24 hours to view or download: USD 50.00

? Add to cart

* Local tax will be added as applicable

\section{Additional information}

\section{Acknowledgement}

We are grateful to Molecular Structure Determination Laboratory, Centre for Research and Instrumentation Management, Universiti Kebangsaan Malaysia (CRIM, UKM) and Atta-urRahman Institute for Natural Products Discovery (AuRIns), Universiti Teknologi MARA (UiTM), Malaysia for NMR analysis. The authors also thank the LCMS Laboratory, Jeffrey Cheah School of Medicine and Health Sciences, Monash University Malaysia, for LCMS analysis. 
People also read

Article

Comprehensive metabolite profiling in distinct chemotypes of Commiphora wightii >
Article

\section{New pyranosyl cembranoid diterpenes from Sarcophyton trocheliophorum >}

Anil Bhatia et al.

Natural Product Research

Volume 33, 2019 - Issue 1

Published online: 2 Feb 2018

Rapid communication

A new cyclohexenone from the tin mine tailings-derived fungus Aspergillus flavus YIM DT $10012>$

Wei-Xun Chunyu et al.

Natural Product Research

Volume 33, 2019 - Issue 1

Published online: 29 Jan 2018

Article

New furostanol glycosides

from Polygonatum

multiflorum (L.) All. >
Mohamed Shaaban et al.

Natural Product Research

Volume 33, 2019 - Issue 1

Published online: 2 Feb 2018

Article

Two new secondary metabolites from a mangrovederived fungus Cladosporium sp. JJM22 >

Jia-Ting Wu et al.

Natural Product Research

Volume 33, 2019 - Issue 1

Published online: 1 Feb 2018
$\alpha$-Glucosidase inhibition by flavonoids: an in vitro and in 

Lii uvacava cuai.

Natural Product Research

Volume 33, 2019 - Issue 1

Published online: 31 Jan 2018 sillico $r$ strucuctúcture-activity Journal of Enzyme Inhibition and Medicinal Eelationship study

Volume 32, 2017 - Issue 1

Published online: 21 Sep 2017

Sample Our

Bioscience journals

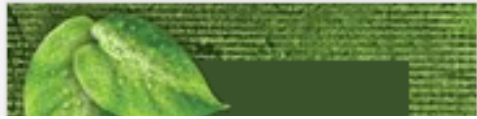




\section{Clinical}

Pharmacognosy

Book Series

\section{Find out more}

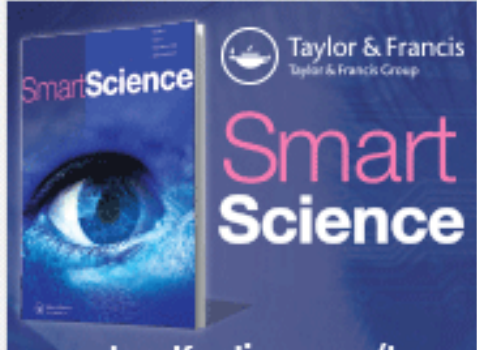

www.tandfonline.com/tsma

\section{Routledge \\ 2. Taylor \& Francis Group}

Routledge Custom

Publishing

Create custom

textbooks with ease 


\section{Information for}

Authors

Editors

Librarians

Societies

Help and info

Help \& contact

Newsroom

Commercial services
Open access

Overview

Open journals

Open Select

Cogent OA

\section{Keep up to date}

Register to receive personalised research and resources by email

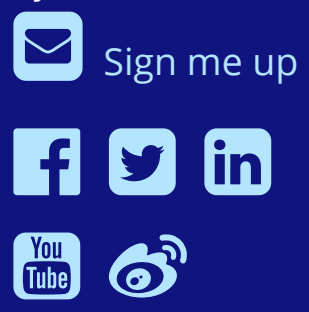

Copyright ( 2018 Informa UK Limited Privacy policy \& cookies Terms \& conditions Accessibility

Registered in England \& Wales No. 3099067

5 Howick Place | London | SW1P 1WG 